\title{
Formación de nanoestructuras orgánicas unidimensionales en superficies semiconductoras*
}

\author{
NOBORU TAKEUCHI**
}

RESUMEN: La formación de nanoestructuras orgánicas autoensambladas (que se forman por sí mismas) sobre superficies de los materiales semiconductores como el silicio es un área de investigación en nanotecnología muy activa e importante. Su estudio es interesante, no sólo por las posibles aplicaciones, sino también desde el punto de vista fundamental. En la investigación básica el interés es motivado por la posibilidad de comprender fenómenos físicos interesantes que están relacionados con estos sistemas, tales como: la estabilidad de las superficies, su crecimiento y las propiedades de los sistemas de una sola dimensión, por mencionar sólo algunos. En cuanto a las aplicaciones tecnológicas, existe un gran interés en el diseño de nanoestructuras orgánicas que combinen las muchas propiedades interesantes de las moléculas orgánicas con la bien desarrollada tecnología del silicio. Esto es particularmente evidente en el campo de la nanoelectrónica, ya que siempre hay una búsqueda para crear circuitos cada vez más pequeños (en la actualidad menor a las decenas de nanómetros), más allá de las capacidades de la litografía normal. Palabras Clave: Nanoestructuras unidimensionales, silicio, superficies, semiconductores

ABSTRACT: The formation of self-assembled organic nanostructures on silicon surfaces is an important and intensely investigated area of nanotechnology. Its study is interesting not only because of possible applications but also from a fundamental point of view. In basic research the interest is motivated by the possibility to understand interesting physical phenomena that are related to these systems, such as surface stability, growth, and the properties of one-dimensional systems, to mention only a few. For technological applications, there is a great interest in the design of organic nanostructures that combine the rich functionalities of organic molecules with the well-developed solid-state semiconductor technologies. This is particularly evident in the field of nanoelectronics, since there is always a search for ways of creating smaller circuits (in the lower tens of nanometers), beyond the capabilities of normal lithography.

\section{INTRODUCCIÓN}

Hay un reciente interés en el estudio de la adsorción de moléculas orgánicas en superficies semiconductoras, no solamente por sus posibles aplicaciones, sino también desde un punto de vista de investigación básica. La introducción de la química orgánica en los semiconductores puede traer nuevas aplicaciones, es por ello que es muy importante el estudio de las reacciones de moléculas orgánicas con las superficies de silicio (Si). La habilidad de este tipo de moléculas para adsorber y emitir luz a ciertas frecuencias, de detectar moléculas, o de reconocer muestras biológicas son ejemplos de algunas aplicaciones que se podrían extender a la tecnología del silicio [1-2].

\footnotetext{
* Agradecimientos: Los cálculos de este trabajo se realizaron en el Centro de Supercómputo de la DGSCAUNAM. Este trabajo fue apoyado por los proyectos Conacyt 48549 y DGAPA-UNAM IN101809.

** Centro de Nanociencias y Nanotecnología, Universidad Nacional Autónoma de México. Ensenada, Baja California, México.
} 
Nanoestructuras orgánicas autoensambladas (que se forman por sí mismas) sobre superficies de materiales semiconductores ya son usadas en aplicaciones prácticas reales, como en pantallas de televisión ultradelgadas y flexibles [3].

Se ha visto que el tipo de crecimiento de estas nanoestructuras puede ser controlado escogiendo cuidadosamente la superficie donde se forman y la molécula que se emplea. Por ejemplo, al usar la molécula de estireno, sobre la superficie hidrogenada de $\operatorname{Si}(111)$ resulta en un crecimiento en dos dimensiones [4-10].

En el caso de la superficie hidrogenada de $\mathrm{Si}(001)$ el depósito de estireno resulta en la formación de nanoestructuras en una dimensión, con una dirección específica (figura 1a). Si se cambia la molécula se puede cambiar la dirección de la formación de la nanoestructura, como se puede ver en la figura $1 \mathrm{~b}$ al depositar moléculas de mercaptán [10].

FIGURA 1. Imágenes de microscopía de efecto túnel donde se muestra la formación de nanoestructuras unidimensionales de moléculas orgánicas en la superficie de $\mathrm{Si}(001)$ (a) estireno y (b) mercaptan. Nótese que dependiendo de la molécula, la orientación de la nanoestructura cambia. Imagen tomada de [10], cortesía de la Dra. Kawai.

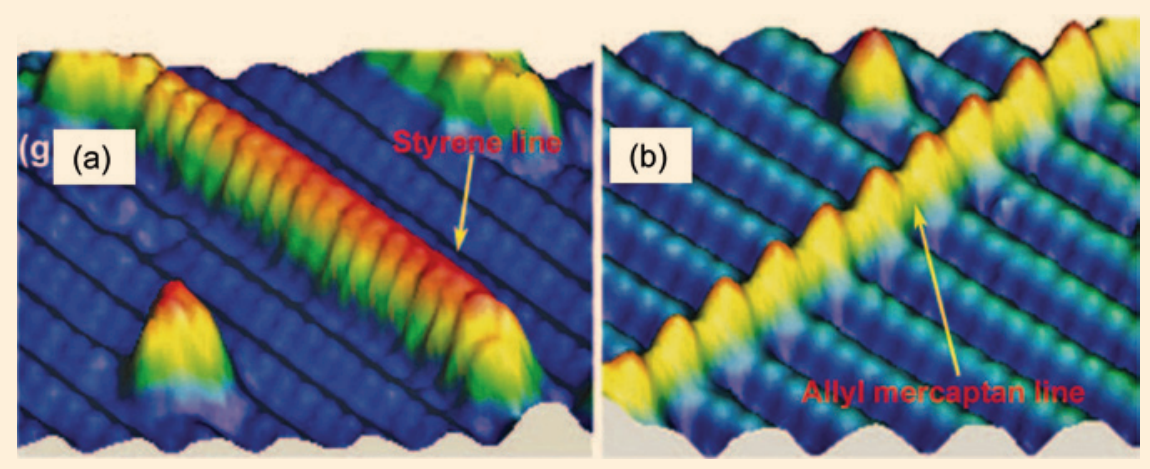

\section{EL SILICIO}

El silicio es el segundo elemento más abundante en el planeta después del oxígeno. Pertenece al grupo IV de la tabla periódica y está debajo del carbono. Tiene un total de 14 electrones y, de ellos, cuatro están en su última capa. Es un material semiconductor, con propiedades intermedias entre los metales y los aislantes [11].

Su estructura cristalina (cuando un sólido tiene una periodicidad en tres dimensiones se dice que es un cristal) es igual a la del diamante y como se puede ver en la figura 2, es cúbica. Como el silicio tiene cuatro electrones de valencia y forma enlaces covalentes, los átomos forman un arreglo tetragonal.

Este tipo de configuración es muy estable en los elementos del grupo cuatro, produciendo enlaces covalentes muy fuertes y es por esto que el diamante (formado por átomos de carbono) es el material más duro que existe en el mundo. 
Figura 2. Estructura cristalina de silicio. Cada átomo de silicio forma tetraedros con sus cuatro vecinos más cercanos.

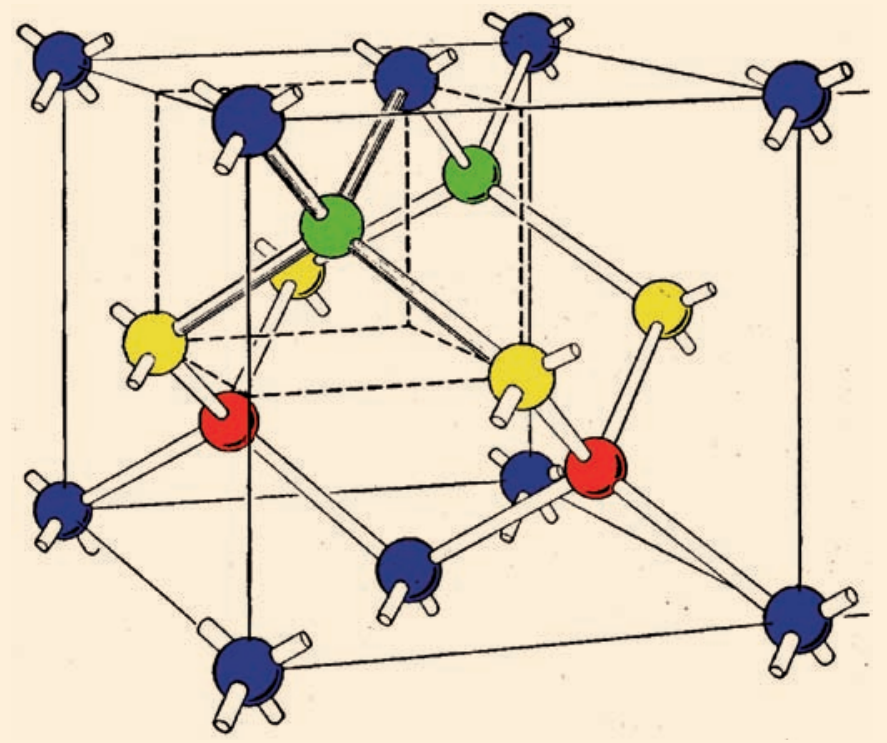

\section{SUPERFICIES DE SILICIO}

Una superficie tiene propiedades distintas a las del sólido cristalino debido a que los átomos en la superficie están en un ambiente diferente (en dos dimensiones) a los átomos del interior, lo que los hace buscar nuevas posiciones de equilibrio. Cuando se crea una superficie de un material como el silicio se forman "enlaces sueltos", los cuales son enlaces químicos asociados a átomos en la capa superficial del sólido que no se une con un segundo átomo sino que se extiende hacia el exterior del sólido. Estos enlaces son energéticamente muy poco favorables y obliga a los átomos en la cercanía de la superficie a reacomodarse para tratar de eliminarlos.

Se pueden obtener diferentes tipos de superficies dependiendo de la manera en que se preparan. Por ejemplo, la superficie formada por los átomos azules de la cara superior del cubo de la figura 2 la llamamos (001). Cada átomo de la superficie está enlazado con dos átomos vecinos de la siguiente capa (círculos verdes en la figura 2) y se queda con dos enlaces "sueltos". Sin embargo, esta situación no es estable energéticamente y los átomos se mueven hasta llegar a la configuración de la figura 3, la cual podemos caracterizar como una dimerización (es decir, la asociación en pares de átomos unidos entre sí) o formación de dímeros. La figura 3b muestra que los dos átomos que conforman un dímero no están a la misma altura vertical y decimos que el dímero es asimétrico. La flecha roja de la figura 3a nos indica una cadena de dímeros asimétricos, en donde podemos observar que la orientación de los dímeros se alterna. 
FIGURA 3. Superficie de silicio (001). Círculos azules denotan átomos de silicio; a) vista superior; b) vista lateral.

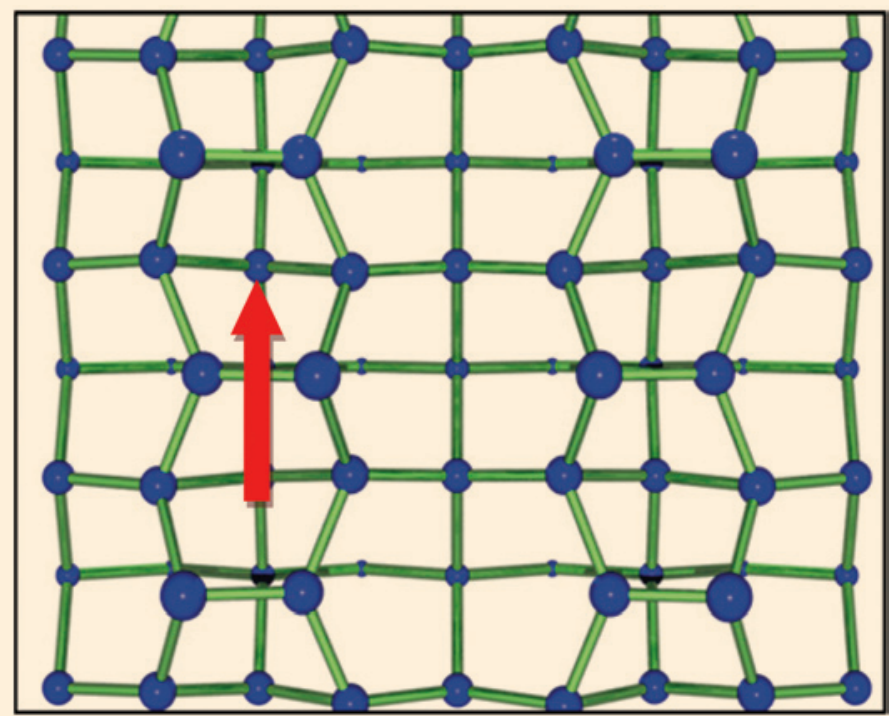

a)

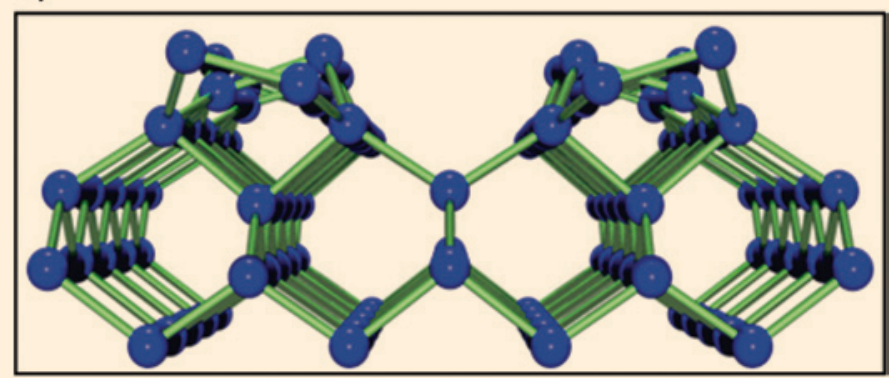

b)

Si a la superficie de silicio (001) se le agrega una capa completa (monocapa) de átomos de hidrógeno, se forma la configuración que se muestra en la figura 4. En esta configuración los dímeros de silicio se mantienen, pero ahora son simétricos. Cada uno de los átomos de la superficie de silicio forma un enlace con un átomo de hidrógeno, completando 4 enlaces: 2 con los átomos de la segunda capa, uno con el otro átomo del dímero y uno con el de hidrógeno. Es por esto que ésta es una configuración muy estable y la superficie no es reactiva. Sin embargo, si existe una vacancia de hidrógeno, se forma un enlace suelto que puede reaccionar fácilmente con moléculas incidentes. Es por esto que esta clase de superficies se usan para la fabricación de nanoestructuras unidimensionales como las de la figura 1. Experimentalmente, estas vacancias se pueden crear usando la punta de un microscopio de efecto túnel. 
FIGURA. 4. Superficie de silicio (001) con una monocapa de hidrógeno. Círculos azules (grises) denotan átomos de silicio (hidrógeno); a) vista superior; b) vista lateral.

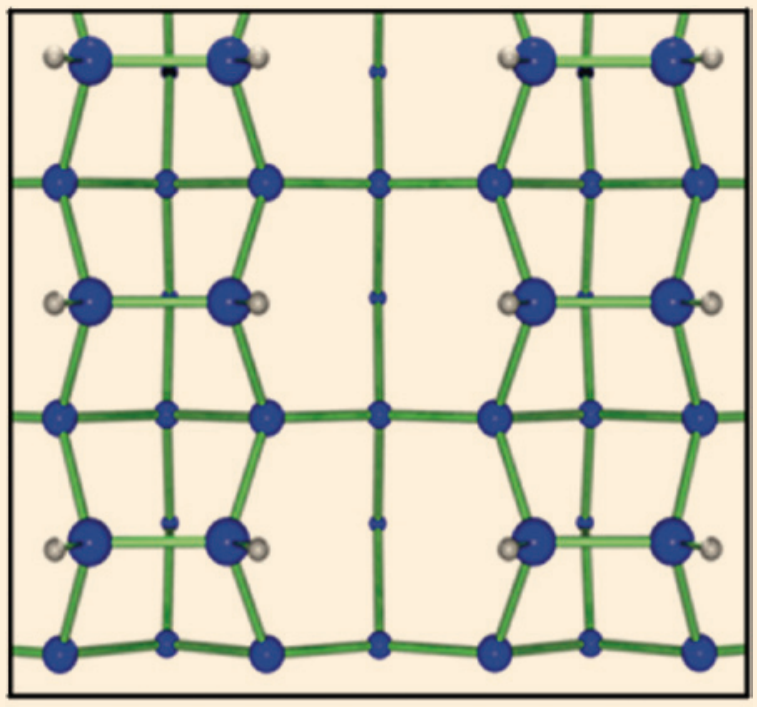

a)

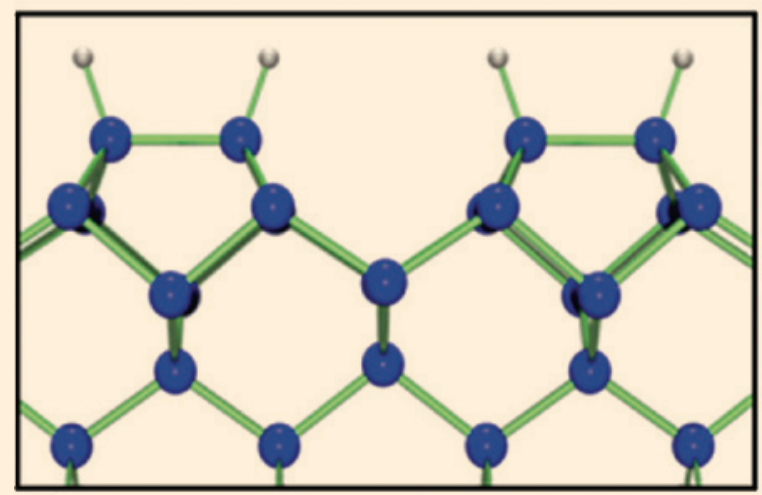

b)

\section{LÍNEAS UNIDIMENSIONALES DE ESTIRENO}

En esta sección vamos a estudiar la formación de nanoestructuras orgánicas unidimensionales sobre la superficie hidrogenada de Si (001). Usaremos como ejemplo el caso de la molécula de estireno; sin embargo, el método puede ser aplicado a cualquier otro tipo de molécula. Experimentalmente, se observa la formación de líneas de estireno paralelas a las cadenas de dímeros de silicio. También se sabe que el crecimiento inicia cuando existe una vacancia de hidrógeno. Actualmente, la única manera para estudiar los mecanismos de formación de estas nanoestructuras unidimensionales a nivel atómico es por medio de simulaciones por computadora. 


\section{Cálculos de primeros principios}

Si al hacer una simulación de un material incluimos desde un inicio datos experimentales para mejorar la concordancia entre los resultados de la simulación y el experimento, estamos hablando de cálculos empíricos y semiempíricos. Otra línea de trabajo consiste en usar sólo la información básica de los átomos (su número y masa atómicos) y estamos hablando entonces de cálculos de primeros principios. Usar métodos empíricos en el estudio de materiales requiere de un menor tiempo de cómputo y se pueden hacer simulaciones con un número mayor de átomos o moléculas. Sin embargo, estos métodos en muchas ocasiones no funcionan, debido a que los parámetros experimentales que se introdujeron en el modelo inicial muchas veces no son muy buenos cuando el sistema que se estudia cambia de configuración.

Por otro lado, los cálculos de primeros principios, al usar solamente el número atómico de los elementos que participan en la simulación y no requerir de ningún tipo de parámetro adicional ni ajuste obtenido con base en resultados experimentales, son muy precisos, además de que pueden hacer predicciones de resultados que no se han obtenido experimentalmente. Sin embargo, tienen la desventaja de que requieren mucho tiempo de cómputo, debido a que usan las leyes de la mecánica cuántica para describir las interacciones de los átomos y los electrones de los materiales que se estudia.

En este trabajo, usaremos la teoría del funcional de densidad que ha sido empleada ampliamente para realizar cálculos de materiales de diferente clase: semiconductores, aislantes y metales. Este método ha mostrado con éxito que se puede transferir de átomos y moléculas individuales a sólidos, superficies y nanoestructuras. Se basa en un teorema que dice que la energía y demás propiedades de un sistema de electrones, en la presencia de un potencial externo, es un funcional único de la densidad de carga [12].

Usando este teorema, podemos optimizar estructuras cristalinas y calcular sus propiedades estructurales y electrónicas, además de su energía total. Esta última nos dice si los sistemas estudiados son estables y si pueden existir. También podemos estudiar las propiedades de sus superficies y en el problema que nos interesa, la reacción de moléculas en las superficies.

\section{Simulación de una reacción química.}

Las reacciones químicas suceden todo el tiempo y en todas partes: al oxidarse un clavo, quemarse un papel, o dentro de nuestro mismo cuerpo (por ejemplo, cuando respiramos).

Sin embargo, la investigación computacional de las reacciones químicas es difícil, ya que a nivel atómico se consideran como eventos "raros": tardan mucho en ocurrir.

Uno de los métodos usados para estudiar computacionalmente una reacción química es la dinámica molecular, en la cual se permite que los átomos o las moléculas interactúen y se muevan por un periodo de tiempo obedeciendo la segunda ley de Newton (fuerza = masa $\mathrm{x}$ aceleración). Aunque en la dinámica molecular las fuerzas de interacción pueden obtenerse empíricamente o por medio de primeros principios, en el estudio de las reacciones químicas es necesario usar estos últimos, ya que una 
reacción química involucra la formación y destrucción de enlaces. El procedimiento para estudiar una reacción por medio de una simulación de dinámica molecular puede sonar muy sencillo: se inicia con los reactantes y se espera un tiempo hasta que reaccionen y se obtengan, finalmente, los productos. Sin embargo, como mencionamos anteriormente, a nivel atómico las reacciones químicas tardan mucho en ocurrir y para la mayoría de reacciones necesitaríamos tiempos extremadamente largos de simulación.

FIGURA. 5. Trayectoria de mínima energía de una reacción química en la que los productos iniciales se transforman en los finales.

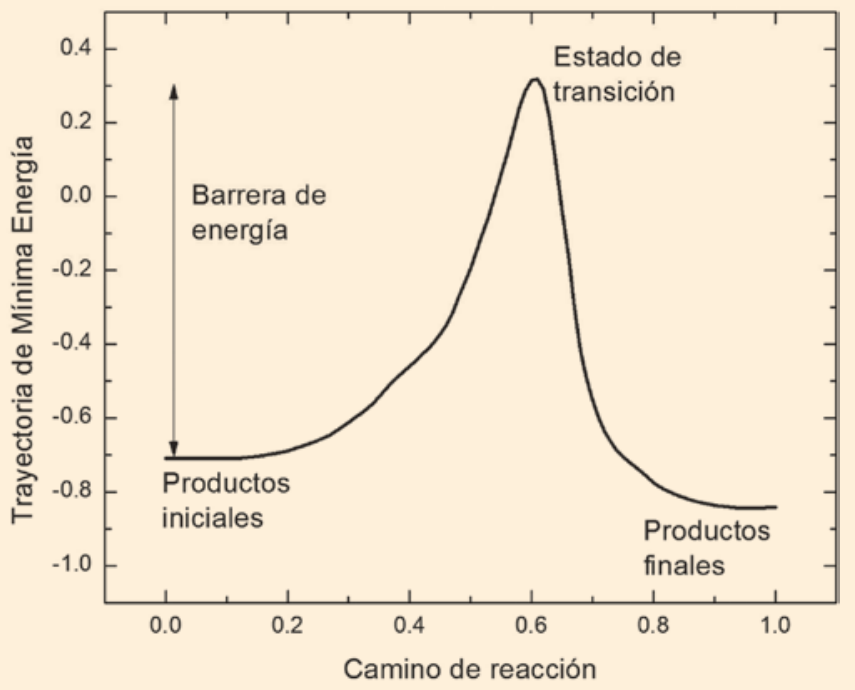

Otro método usado es la teoría del estado de transición. En ella se parte de un estado inicial de los reactantes y se calcula la trayectoria de mínima energía (TME) para llegar a los productos, como se muestra en la figura 4. El estado de transición en una reacción química es una configuración particular a lo largo de la TME que corresponde al máximo de energía. En este punto se asume que las especies reactantes conducirán siempre a la formación de los productos. La diferencia de energías entre los estados de transición e inicial es la barrera de energía que se debe de vencer para que la reacción se pueda completar. Dependiendo del tamaño de las barreras de energía, podemos saber si una ruta de reacción es posible o no.

\section{Molécula de estireno}

El estireno (figura 6) es una molécula orgánica con fórmula $\mathrm{C}_{6} \mathrm{H}_{5} \mathrm{CH}=\mathrm{CH}$, también se conoce como vinil benceno porque está formado por un benceno pegado a un grupo vinilo (con un doble enlace $\mathrm{C}-\mathrm{C}$ ). 
FIGURA. 6. Molécula de estireno. Círculos rojos y grises representan átomos de carbono e hidrógeno, respectivamente.

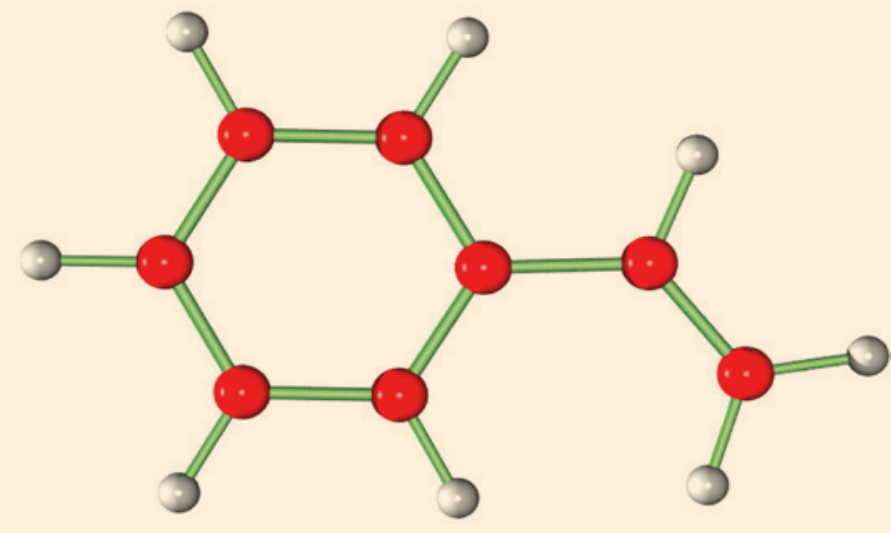

\section{Adsorción de una molécula de estireno sobre la superficie hidrogenada}

El proceso de la reacción de la molécula de estireno con la superficie hidrogenada de $\mathrm{Si}(001)$ se puede ver en la figura 7. Inicialmente, la molécula de estireno se pega a la superficie de silicio en la vacancia de hidrógeno, saturando el enlace suelto de silicio (figura 7a). En ese estado, el estireno forma lo que en química se conoce como radical. No es una molécula estable, porque se rompe parcialmente el enlace doble de C-C. Debido a que en esta situación queda un electrón sin aparear, este radical es muy reactivo y trata de "robar" un átomo de hidrógeno de algún sitio vecino.

Hay dos posibilidades: que el hidrógeno provenga de la misma cadena de dímeros de silicio, o que sea tomado de la cadena vecina. En el primer caso se tiene la formación de una nanoestructura paralela a las cadenas de silicio, mientras que en el segundo caso es perpendicular. Los cálculos de las trayectorias de mínima energía para estos procesos indican que la barrera de energía para completar este proceso (la figura 7b muestra la geometría en la cima de la barrera, o sea, el estado de transición) es más pequeña en el primer caso, indicando que el crecimiento de las nanoestrucutras es paralelo a las cadenas de silicio como se observa en el experimento. La curva azul en la figura 7 muestra la trayectoria de mínima energía cuando el átomo de hidrógeno se transfiere de un sitio en la misma cadena de dímeros de silicio.

Una vez que se tiene esta transferencia del átomo de hidrógeno, la molécula se vuelve estable y además se tiene un nuevo enlace suelto en el átomo de silicio vecino como se observa en la figura 6c (el enlace suelto se muestra como un óvalo azul). Los siguientes procesos de la reacción son dos rotaciones sucesivas mostradas en las figuras $7 \mathrm{~d}$ y $7 \mathrm{e}$. Al final de este proceso se tiene una molécula estable adsorbida en la vacancia de hidrógeno original, mientras se forma una nueva vacancia de hidrógeno en el sitio vecino, donde se puede pegar una nueva molécula de estireno y repetir el proceso descrito anteriormente hasta formar cadenas muy largas de estireno sobre la superficie de Si (001) como se muestra en la figura 8. 
FIGURA. 7. Vista lateral de los pasos en la reacción de la molécula de estireno con la superficie de Silicio (001) con una monocapa de hidrógeno. Círculos azules, grises y rojos denotan átomos de silicio, hidrógeno y carbono, respectivamente.
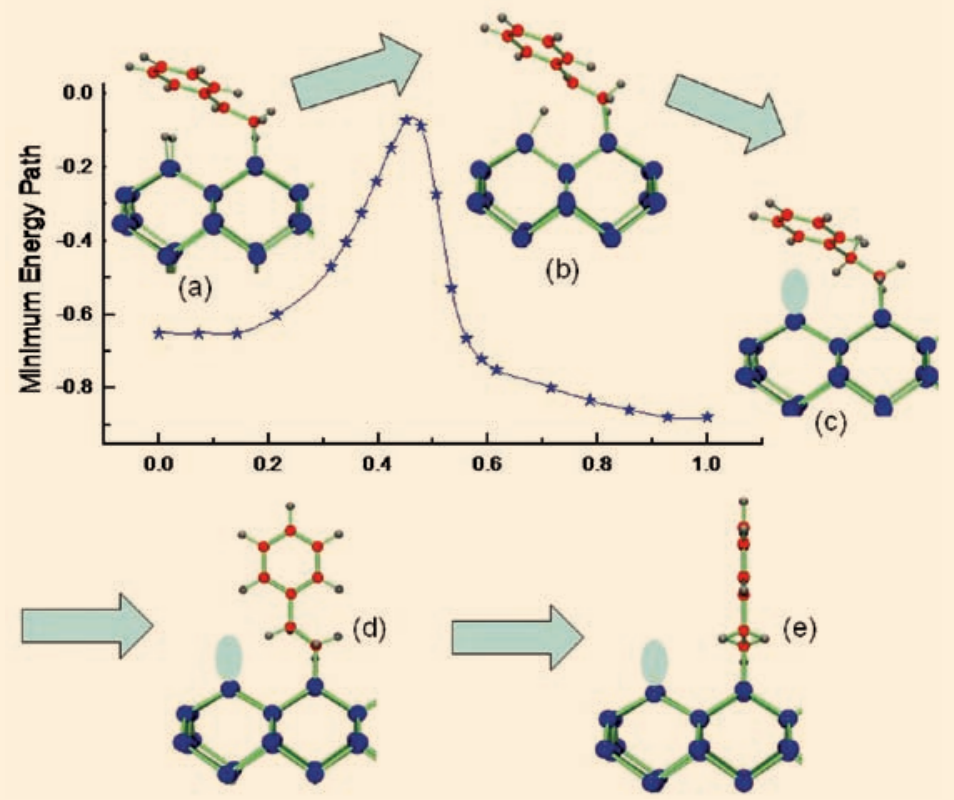

FIGURA. 8. Nanoestructura unidimensional formada por el estireno en la superficie de Silicio (001).

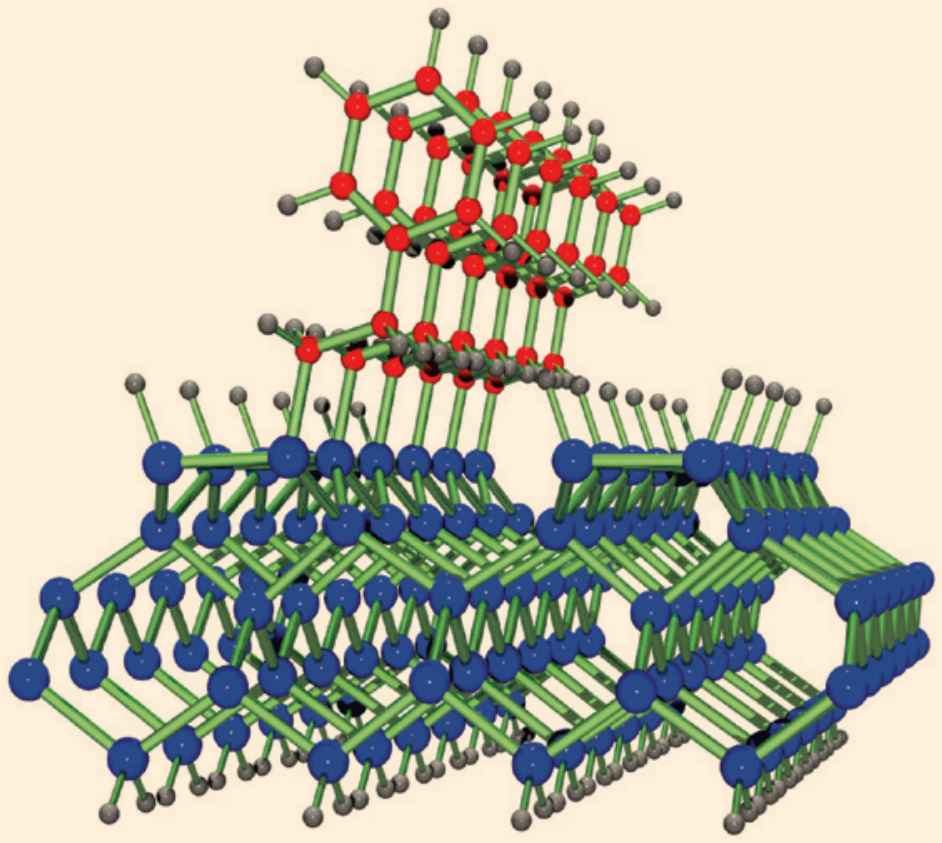




\section{RESUMEN}

Moléculas orgánicas pueden formar nanoestructuras autoensambladas en las superficies de silicio. Estos estudios son importantes por razones tanto prácticas como de investigación básica. En el primer caso, se buscan combinar las propiedades de la química orgánica con la tecnología del silicio, para aumentar así, las posibles aplicaciones de este importante material semiconductor. Desde el punto de vista de la investigación básica nos permite entender fenómenos físicoquímicos interesantes que ocurren en sistemas de dos y una dimensión. En particular, mostramos que el estireno forma nanoestructuras unidimensionales sobre la superficie (001) de silicio con una monocapa de hidrógeno. La reacción química comienza en una vacancia de hidrógeno, y las nanoestructuras se forman paralelamente a las cadenas de dímeros de silicio.

\section{Referencias}

[1] Buriak, J. M. 2002. Chemical Reviews, 102: 1271-1308.

[2] Bent, S. F. 2002. Surface Science, 500: 879-903.

[3] Moller, S.; Pelov, C.; Jackson, W.; Taussig, C.; and Forrest, S.R. 2003. Nature, 426: 166.

[4] Cicero, R.L.; Chidsey, C.E.D.; Lopinsky, G.P.; Wayner, D.D.M.; Wolkow, R.A. 2002. Langmuir, 18, 305.

[5] Takeuchi, N; Kanai Y.; Selloni A. 2004. Journal of the American Chemical Society, 126, 15890.

[6] Tong, X.; DiLabio G. A.; Wolkow, R.A. 2004. NanoLetters 4: 979.

[7] Lopinsky, G.P.; Wayner, D.D.M.; Wolkow, R.A. 2000. Nature, 2000, 406: 48-51.

[8] Takeuchi, N; Selloni, A. 2005. Journal of Physical Chemistry B. 10911967.

[9] Takeuchi, N; Selloni, A. 2010. Journal of Physical Chemistry C, 114, 3981.

[10] Hossain MZ, Kato HS, Kawai M. 2005. Journal of the American Chemical Society, 127, 15030.

[11] Herrera-Zaldívar M, Valenzuela-Benavides J. 2008. Mundo Nano, 1, 13.

[12] Takeuchi, N. 1998. Ciencia y Desarrollo, 24, 18. 